\title{
Analysis and prediction of building damage due to windstorms
}

\author{
N. O. Nawari \\ School of Architecture, College of Design, Construction and Planning, \\ University of Florida, Gainesville, USA
}

\begin{abstract}
It's been more than five years since a major storm hit a major US city, however hurricane researchers have estimated that the next one could cause as much as $\$ 150$ billion worth of damage.

An understanding of the damage mechanisms due to a variety of natural hazards such as wind, storm surges, and tsunamis windstorm hazard is integral to determining the best design and construction practices. Therefore, an anatomical analysis of a large suite of damaged structures in similar extreme events, such as Florida coastal areas, promises to reveal causes of failure in coastal construction and how best to prevent similar damage in the future.

Prediction and classification of hurricane buildings damage involves many sources of uncertain data that make it a difficult task using conventional prediction models. This work seeks to elucidate the application of data mining algorithms in the prediction and classification of damage due to hurricane and tornadoes forces. The research focuses on the conceptual and applied frameworks for the data mining models to assist in the prediction, assessment, and classification of buildings damages caused by server windstorms. Keywords: wind storms, building damages, prediction, data mining.
\end{abstract}

\section{Introduction}

High winds, airborne projectiles, wind-driven water, sea surges, and flooding are among the hazards that threaten buildings and their occupancies. The record for US property insurance tells the story. Annual claims for wind damage claims tally hundreds of millions of dollars. Florida homeowners pay some of the nation's highest insurance premiums; in a recent poll, despite a housing crisis, an 
economic crisis, a water crisis and an environmental crisis, Floridians named those premiums their number-two concern about the state's future, behind property taxes but ahead of jobs, education, health care and the dying Everglades.

It's not that coastal areas vulnerability is a secret. Continued development of coastal areas without consideration to the range of natural hazards, and the benefits and consequences of measures taken to mitigate them, will undoubtedly result in future storm and flood damages, and increased public expenditures for post-storm response and recovery.

Since Hurricane Andrew put most Florida insurers out of business and scared several national insurers out of the state, the state government has helped to hedge the risk of hurricanes. It provides subsidized insurance to 1.3 million highrisk homeowners who can't get private policies, an increase of more than $50 \%$ in just three years. It also has a Hurricane Catastrophe Fund that provides subsidized reinsurance to the state's private firms. But no one denies that the risk is real: it's been 80 years since a major storm hit a major Florida city, but hurricane researchers have calculated that the next one could cause as much as $\$ 150$ billion worth of damage.

According to NIST technical note 1476, at the time of hurricane Katrina, there was no statewide building code in Louisiana, Mississippi, Alabama, or some parts of Texas, although some local jurisdictions within those states had adopted model building codes. The City of New Orleans had adopted the 2000 edition of the model building and residential codes issued by the International Code Council in January 2004. Significant damage was observed in many instances where the winds were lower than those levels cited in codes and standards suggesting that the there are many uncertainties involved in structural systems subjected to hurricane forces and illustrating the inadequacy of our current approaches. Thus, an understanding of the damage mechanisms due to a variety of natural hazards such as wind, storm surges, and tsunamis windstorm hazard is integral to determining the best design and construction practices. Therefore, an anatomical analysis of a large suite of damaged structures in similar extreme events, such as Florida coastal areas, promises to reveal causes of failure in coastal construction and how best to prevent similar damage in the future.

\section{Anatomy of windstorm}

Windstorm is a very complicated phenomenon. It is air and water in turbulent flow, which means that the motion of individual air or water particles is so erratic that in studying storm one ought to be concerned with statistical distributions of speeds and directions rather than with simple averages or fixed physical quantities. For analytical model, storm forces can be classified as one of a combination of:

- Wind Pressure

- Windborne Debris

- Falling objects 
- Flood Pressure

- Rain Forces

The resistance of buildings to wind pressures has been the subject of considerable research and is addressed by building codes. However, normal design loads specified in these codes are substantially lower than those that occur during a windstorm. This is due to many sources of uncertainties involved in the computational model for the forces mentioned above. For instance, recent studies (Mosqueda and Porter [8]) have shown that damage due to Katrina hurricane extended beyond residential communities, with significant damage to engineered infrastructure, including buildings, roads and bridges, utility distribution systems for electric power and water, wastewater collection facilities, and vital communication networks, which are designed using standard design loads for gravity and lateral resisting elements. The ASCE 7 provision describes computational method for wind pressure using a number of coefficients that require considerable judgment to determine which pressure coefficients to use, how to determine tributary areas for cladding and framing elements, and whether building elements should be designed as part of the main wind force resisting system or components and cladding. The corners, edges, and eave overhang of a building are subjected to complicated forces as windstorm passes these obstructions, causing higher localized suction forces that are not considered appropriately in Building Codes. Moreover, there is no computational model or standard test protocol in the industry for the critical structural elements that addresses storm pressures generated by hurricanes or tornadoes.

\section{Damage prediction}

Buildings respond to windstorm forces in a variety of complex and dynamic ways. The windstorm destructions seems to be an intricate phenomenon associated with multidimensional uncertain dataset that is difficult to analyze entirely using classical approach.

Different researchers employed different methods to assist in the prediction of hurricane damages. For instance, Sill and Kozlowski [9] presented a method for predicting the percentage of damage within an area as a function of wind speed and various other parameters. The practicality of the proposed method is hampered by insufficient clarity and transparency. Huang et al. [2] presented a risk assessment strategy based on an analytical expression for the vulnerability curve. The expression is obtained by regression techniques from insurance claim data for hurricane Andrew. Khanduri and Morrow [5] also presented a similar method of damage assessment. Although such approaches are simple, they are highly dependent on the type of construction and construction practices common to the areas represented in the claim data. Burrus et al. [1] estimated the impact of low-intensity hurricanes as 'business interruption' of regional economies in North Carolina. A hurricane wind damage prediction model that incorporates a time-stepping component-based Monte Carlo simulation approach is being implemented for the FEMA HAZUS project (Lavelle et al. [6]). Hazards United States-Multi-hazard _HAZUS-MH, the FEMA hazard prediction program 
developed under contract with the National Institute of Building Sciences, uses GIS software to map and graphically depict hazard data, economic losses, and buildings and infrastructure damage from hurricanes, floods, and earthquakes. The system applies both loss estimation and risk assessment methodologies for a limited set of disasters. Although HAZUS-MH is equipped to model the probability and level of pre-incident risk, the system can neither support realtime response and recovery nor building information modelling (BIM) platform. Some studies also reported a loose prediction when compared with real data. Furthermore, using HAZUS to predict loss from a hurricane requires expert skills in meteorology to validate the input data used to develop the hurricane scenario and expert skills in wind and forensic engineering to assess the effectiveness and accuracy of the model output.

The hurricane damages seems to be a complex phenomenon associated with multidimensional uncertain data that is difficult to analyze entirely using classical approach. Data mining analysis offers an important tool in understanding and capturing details relationship between the different parameters involved.

\section{Data mining analysis}

Data mining is the process of extracting valid, authentic, and meaningful relationships from large quantities of data. It involves uncovering patterns in the data and is often tied to data warehousing because it attempts to make large amounts of data actionable.

Data elements fall into distinct categories; these categories enable making predictions classifications about other pieces of data. For example, building damages can be assessed from a large amount of data about the building sizes, location, geometric shapes, main materials, gravity and lateral resistive systems, and the intensity of storm. Knowing this basic building information, engineers can use data mining to models predictions about the expected degree of damages. One of the more difficult aspects of applying data mining in engineering practice has always been translating the theory into routine techniques.

A fundamental concept is that building a mining model is part of a larger process that includes all from defining the basic problem that the model will solve, to deploying the model into a working environment. This process can be defined by using the following basic steps: (i) define the problem, (ii) preparing data, (iii) defining models, (iv) validation and exploration, (v) deploying and updating models. The following diagram shows the process involved in the datamining analysis:

The first step includes analyzing the requirements, defining the scope of the problem, defining the metrics by which the model will be evaluated, and defining the final objective for the data mining project. These tasks can be summarized in the following: Defining the datasets for the analysis, Identifying the attributes of the dataset that we want to try to predict, What pattern and associations are we seeking? 
The second step involves the preparation, which may include calculating the minimum and maximum values, calculating mean and standard deviations, and looking at the distribution of the data. Microsoft SQL2008 server has a data Source View Designer in Business Intelligence (BI) Development Studio that contains several tools that allows such data exploration.

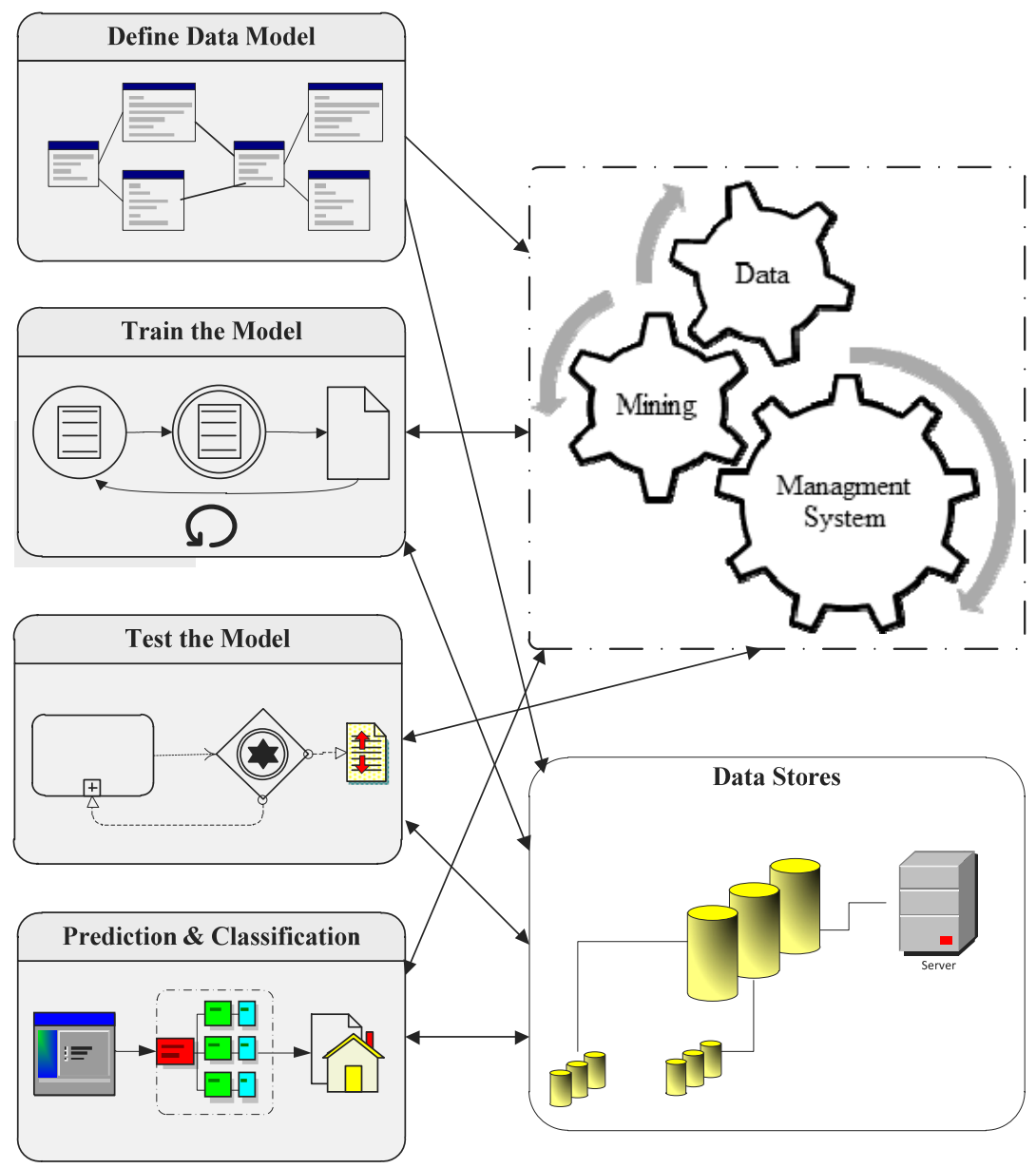

Figure 1: Data mining analysis process.

Before starting the analysis, the model data must be randomly separate into training and testing datasets. This step can be achieved by using the Percentage Sampling Transformation service available with SQL 2008 server as a part of the Integration Services. The Percentage Sampling Transformation creates a sample dataset by selecting a percentage of the transformation input rows. The sample dataset is a random selection of rows from the transformation input, to make the 
resultant sample representative of the input. The training dataset is utilized to build the model, and the testing dataset to verify the accuracy of the model.

A data mining model is typically defined by specifying input columns, an identifying column, and a predictable column. Alternatively, once can define these columns in a new model by using the Data Mining Extensions (DMX), or the Data Mining Wizard in BI Development Studio. This is known as mining structure that defines the data domain from which mining models are built. A single mining structure can contain multiple mining models that share the same domain. This structure contains information such as data type, content type, and how the data is distributed.

After defining the structure of the mining model objects, training starts by populating the empty structure with the patterns that describe the model. Patterns are found by passing the original data through a mathematical algorithm. SQL Server 2008 contains different algorithms. The data mining algorithm is the mechanism that creates mining models. To create a model, an algorithm first analyzes a set of data, looking for specific patterns and trends. The algorithm then uses the results of this analysis to define the parameters of the mining model.

In summary, a mining model is defined by a data mining structure object, a data mining model object, and a data mining algorithm. Microsoft SQL Server 2008 Analysis Services (SSAS) provides several algorithms for use in data mining solutions: Decisions Trees, Clustering, Association Rules, Naïve Bayes, and Neural Network.

\section{Prediction}

Most of the building damage predictions have been too focused on treating single hazards in isolation and must be broadened to include the capacity to account for multiple hazards. The multi-hazard approach takes into account the mitigation strategies for various hazards simultaneously within a balanced approach. The first step in multi-hazard prediction is to determine the risks that are specific to a structure's site. The site must be analysed based on geographic location and previous history. The loads, building type, shapes, size and form, degree of exposure and resulting structural response associated with each potential hazard a structure may encounter must be described and quantified in the data model. Knowledge of the damage mechanisms is imperative in determining how hazards relate to each other and what aspects of the building are particularly affected. These data are partially described in the database structure depicted in figure 2 below.

The relationship between geographic locations, form, sizes and geometric shapes of concrete, steel, timber and masonry residential building including their primary gravity and lateral resistive systems, the intensity and type of storms, and the degree of damages can be analyzed using the data mining models techniques suggested herein to provide supportive damage prediction system. 


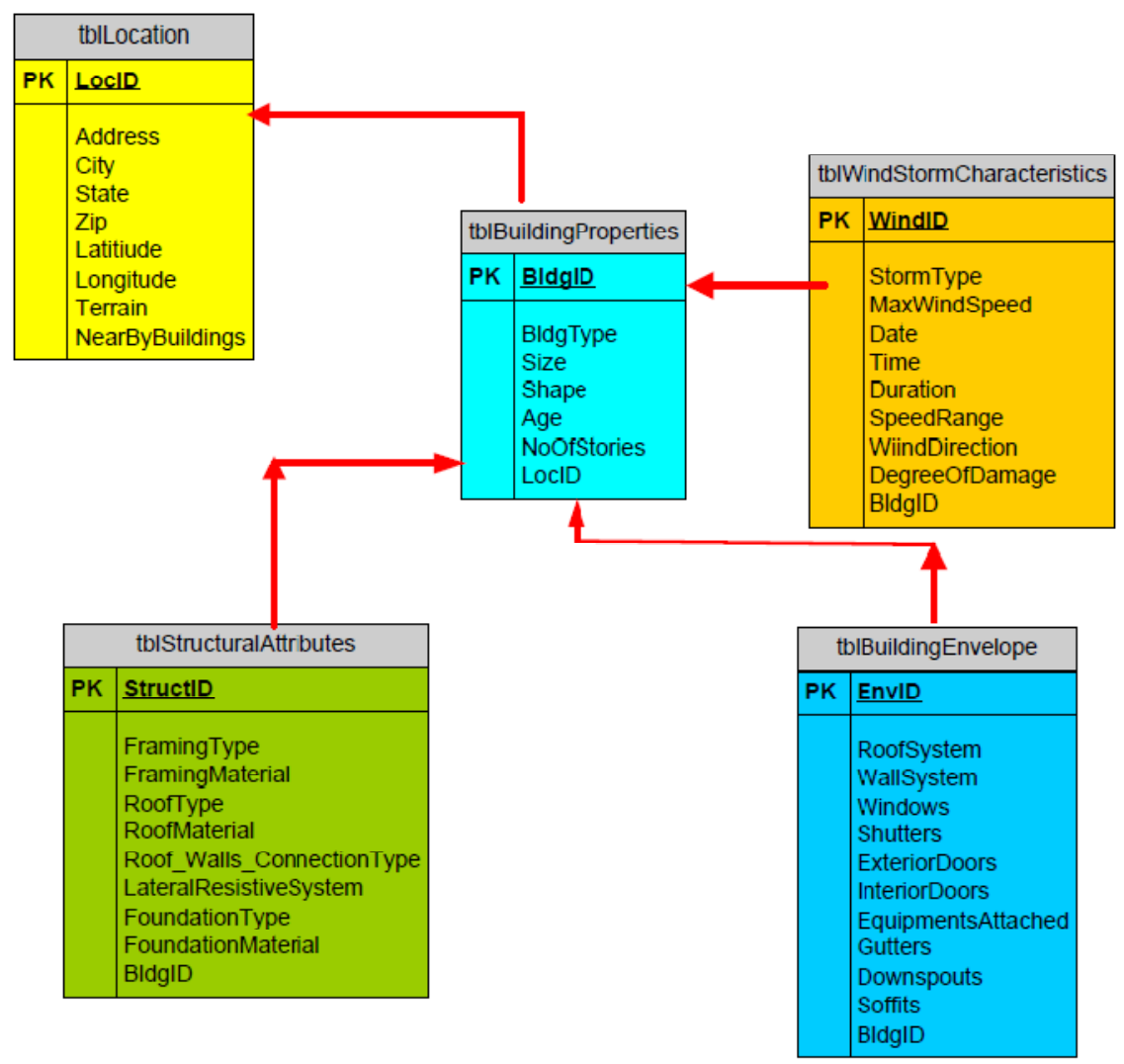

Figure 2: $\quad$ Part of the database diagram for the data mining analysis.

The output prediction vector is based on the damage categories specified by FEMA320. These categories are modified to include the distinction between envelope and structural damages of buildings. In contrast to FEMA320, ten damage categories are proposed in this study.

(1) Minimal: No real structural damage is done. Minor building envelope damages may occur (less than 5\%).

(2) Low Moderate: 6\%-10\% Roof and other envelope components are damaged.

(3) Moderate: 11\%-20\% Roof and other envelope components are damaged.

(4) High Moderate: more than 20\% Roof and other envelope components are damaged.

(5) Low Extensive: less than 10\% Structural damage is done along with damages to envelope.

(6) Extensive: less than 20\% Structural damage is done along with damages to envelope. 
(7) Low Extreme: Extensive damage is done to many envelope components (21\%-30\%) accompanied by structural damages $(21 \%-30 \%)$

(8) Extreme: Extensive damage is done to many envelope components $(31 \%-50 \%)$ accompanied by structural damages $(31 \%-50 \%)$ that may result in complete building failure.

(9) Very Extreme: Extensive damage is done to many envelope components $(51 \%-60 \%)$ accompanied by structural damages $(51 \%-60 \%)$ that may result in complete building failure.

(10) Catastrophic: Envelope damage is extensive and widespread ( $>60 \%)$. Structural damages considerable and there are complete to near complete buildings failure.

Many researchers have noted that code-based design, as it exists today, may not be sufficient in many coastal areas (Kareem [4], Lee and Rosowsky [7]). Instead, performance-based design is receiving attention in areas affected by multiple hazards. Avoiding collapse and maintaining occupant safety may not be the only expectations. Therefore, the design process must incorporate the relationship between performance and global risk of failure. Exceeding the requirements of local and national building codes may increase initial construction costs, but long-term benefits in overall robustness and resiliency of a structure may easily outweigh these initial costs.

\section{Conclusions}

Code-based building design, as it exists currently, may not be sufficient in the United States Gulf and Atlanticcoastal areas. These areas where most of the country's windstorm related fatalities have occurred are also now experiencing the country's most significant growth in population and buildings. Under these conditions, multiple hazards with which a building may be faced must be considered in all aspects of design and construction. A multi-hazard design approach would ensure that design strategies used to mitigate one hazard would not affect the structures ability to resist another hazard. The probabilities of failure and consequences of failure can be compared to determine the best way to lower the overall risk of failure. An anatomical data mining analysis will provide such information and assist to design strong, multi-faceted structural systems that mitigate damage from many hazards.

The relationship between windstorm and building damages sited raises many uncertainties about current building design and construction practices. Application of data mining analysis provide a supportive tool to handle uncertainty and discover hidden relationships and rules that assist in classifying, predicting and associating multihazard building damages and windstorm patterns. The system could also be instrumental in updating Building Codes and standard. Buildings that use multihazard engineering strategies in their initial design stages will be able to withstand loads resulting from various circumstances and will maintain structural integrity for years longer than traditionally designed buildings. From the severity of destructions shown in 
recent hurricanes and tornadoes, it is apparent that Building Codes and Standards need to re-address windstorm resistive systems.

\section{References}

[1] Burrus, Robert, Christopher F. Dumas, Claude H. Farrell and William W. Hall, Jr. "Impact of Low-Intensity Hurricanes on Regional Economic Activity", Natural Hazards Review, pp 118-125, August 2002.

[2] Huang, Z., Rosowsky, D. V., and Sparks, P. R. "Long-term hurricane risk assessment and expected damage to residential structures." Reliability Eng. Sys. Safety, 74, 239-249, 2001.

[3] HAZUS -MH_. "HAZUS-Multi disasters, a loss estimation software." http://www.fema.gov/hazus/hz_overview.shtm, 2011.

[4] Kareem, A, "Structural Performance and Wind Speed Damage Correlation in Hurricane Alicia", Journal of Structural Engineering, Volume 111, Issue 12, pp. 2596-2610, December 1985.

[5] Khanduri, A. C., and Morrow, G. C. "Vulnerability of buildings to windstorms and insurance loss estimation." J. Wind. Eng. Ind. Aerodyn., 91, 455-467, 2003

[6] Lavelle, F., Vickery, P. J., Schauer, B., Twisdale, L. A., and Laatsch, E. "The HAZUS-MH Hurricane Model." Proc., 11th Int. Conf. on Wind Engineering, Texas Tech Univ., Lubbock, Tex, 2003.

[7] Lee, K.H., and D.V. Rosowsky, "Fragility analysis of woodframe buildings considering combined snow and earthquake loading", Structural Safety, 28, pp. 289-303, 2006.

[8] Mosqueda, G. and K.A. Porter, "Preliminary conclusions: assessing damage to engineered buildings in the wake of Hurricane Katrina." Structural Engineer, 20-26, February 2006.

[9] Sill, B. L., and Kozlowski, R. T. "Analysis of storm damage factors for low-rise structures.” J. Perform. Constr. Facil.,11(4), 168-177, 1997. 